\title{
Electric-field manipulation of spin states in confined non-magnetic/magnetic heterostructures
}

\author{
S Borza ${ }^{1,2,3}$, F M Peeters ${ }^{1}$, P Vasilopoulos ${ }^{1,4}$ and G Papp $^{1,2,3}$ \\ ${ }^{1}$ Departement Fysica, Universiteit Antwerpen (Campus Groenenborger), Groenenborgerlaan 171 \\ B-2020 Antwerpen, Belgium \\ 2 Department of Theoretical Physics, University of Szeged, Aradi vtk. tere 1, H-6720 Szeged, \\ Hungary \\ ${ }^{3}$ Institute of Physics, University of West Hungary, Bajcsy Zsilinszky út 4-6 H-9400 Sopron, \\ Hungary \\ ${ }^{4}$ Department of Physics, Concordia University, 1455 de Maisonneuve Ouest Montréal, QC, \\ H3G 1M8, Canada \\ E-mail: borzas@fmk.nyme.hu,francois.peeters@ua.ac.be, takis@alcor.concordia.ca \\ (P Vasilopoulos) and pgy@physx.u-szeged.hu
}

Received 12 October 2006, in final form 7 March 2007

Published 10 April 2007

Online at stacks.iop.org/JPhysCM/19/176221

\begin{abstract}
The energy spectrum and states of an electron in a non-magnetic/magnetic heterostructure placed between two materials (e.g. oxides) acting as barriers is studied in the presence of a magnetic field perpendicular or parallel to the well. A potential step is formed at the interface between the non-magnetic and magnetic material in the presence of a magnetic field since spin-up electrons see a barrier whereas the spin-down ones see a well. A rich band structure is obtained which can be tuned by a perpendicular electric field. Numerical results are presented for a $\mathrm{ZnSe} / \mathrm{Zn}_{1-x} \mathrm{Mn}_{x}$ Se heterostructure and their pertinence to spin-polarized transport is pointed out.
\end{abstract}

\section{Introduction}

With the emergence of the field of spintronics the study of magnetic quantum structures has been intensified due to their potential usefulness in a new generation of devices that exploit the spin rather than the charge of the electrons [1]. Some of the structures studied involve inhomogeneous magnetic fields [2], on the nanometre scale, either external or built-in as in the case of diluted magnetic semiconductors (DMS) (see [3] for recent reviews). More specifically, magnetic resonant tunnelling diodes [4] and spin filters [5, 6] have been studied as well as spin transport in asymmetric tunnel structures [7], spin-polarized ballistic transport in a quantum wire [8], magnetic-field switching of spin injection and coherence [9], energy transfer in ZnSebased double quantum wells [10], etc. In addition, Monte Carlo studies of magnetic ordering have been reported [11], and more recently the influence of positional disorder of $\mathrm{Mn}$ atoms 


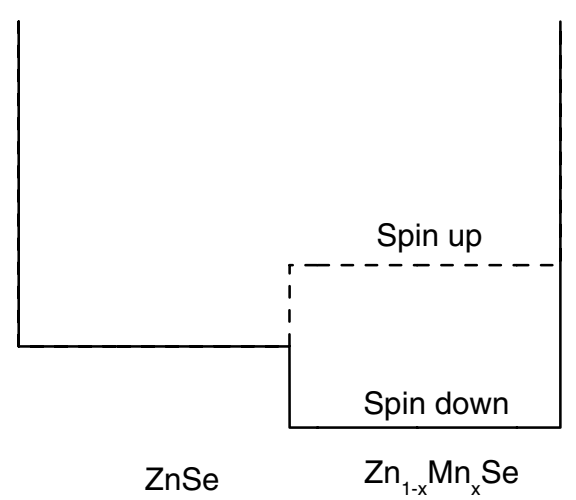

Figure 1. Schematics of the structure: a non-magnetic/magnetic heterostructure is confined by infinitely high potential walls. It has a step at the interface between the non-magnetic ( $\mathrm{ZnSe}$ ) and magnetic $\left(\mathrm{Zn}_{1-x} \mathrm{Mn}_{x} \mathrm{Se}\right)$ material when an external magnetic field is applied.

on the magnetic properties has been investigated $[12,13]$. Many more topics, including spinpolarized transport, can be found in the very recent review of [16].

Experimentally it has been shown that in a GaAs:Mn DMS quantum well it is possible to manipulate the magnetization state, e.g. to reverse it, using a perpendicular electric field [17]. The mechanism for this reversal is based on a variation of the hole density induced by the electric field which in turn changes the Curie temperature. In the present study we consider a II-VI DMS system where the free carriers are electrons instead of holes and the manipulation of the magnetic state is due to an electric-field induced spatial shift of the electron wavefunction at constant electron density. In detail, we study the energy spectrum and states of an electron in a quantum well formed when a non-magnetic/magnetic heterostructure is

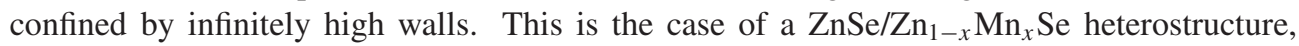
confined between, e.g., oxide layers, and the resulting quantum well has a step at the interface between $\mathrm{ZnSe}$ and $\mathrm{Zn}_{1-x} \mathrm{Mn}_{x} \mathrm{Se}$, as shown in figure 1 for the case in which the band offset between $\mathrm{ZnSe}$ and $\mathrm{Zn}_{1-x} \mathrm{Mn}_{x}$ Se is negligibly small $[4,14,15]$. Our study takes into account a magnetic field perpendicular or parallel to the well, as well as an electric field perpendicular to the well. Usually the effect of the positional disorder of Mn atoms on the subbands is neglected $[4,14,15]$. Though for low concentrations $x$ this disorder can have an important effects on the subbands and the magnetic properties $[12,13]$, we will not include it in our study.

In the next section we formulate the problem and derive the corresponding differential equations that we solve numerically. In section 3 we present numerical results for the energy levels, electron velocity and density of states (DOS). Then in section 4 we discuss the tunability of the polarization of the two-dimensional electron gas (2DEG) in the quantum well by a perpendicular electric field and make concluding remarks in section 5.

\section{Formulation of the problem}

We consider electrons in the non-magnetic/magnetic quantum well shown in figure 1 . In order to reduce the number of parameters we assume for convenience that the quantum well is formed by infinitely high barriers. The generalization to barriers of finite height is straightforward but it will not change qualitatively the physics we will discuss in the present work.

In Mn-based semi-magnetic II-VI semiconductor systems in the presence of an external magnetic field conduction electrons interact with the $3 \mathrm{~d}$ electrons of the localized magnetic 
moments of the Mn ions via the sp-d exchange interaction. As a result, spin-up electrons see a barrier while spin-down electrons see a well, and a stair potential well results as shown in figure 1. Within mean field theory and for a magnetic field $B$ along the $z$ axis, the sp-d exchange interaction gives rise to the potential $V_{\sigma_{z}}=-N_{0} \alpha \sigma_{z} x_{\text {eff }}\left\langle S_{z}\right\rangle$ in the Mn-doped layer, while $V_{\sigma_{z}}=0$ in the ZnSe layer. Here, in line with [5, 14] and [15], we neglect a conduction band offset between the two layers. A similar potential arises when $B$ is perpendicular to the well but in this case the potential depends on the electron spin component along the $y$ direction. Here $N_{0} \alpha$ is the electronic sp-d exchange constant, $\sigma_{z}= \pm 1 / 2$ the spin component parallel to the field $B, x_{\text {eff }}=x(1-x)^{12}$ is the effective Mn concentration accounting for antiferromagnetic pairing [5,9], $x$ is the real Mn concentration, $\left\langle S_{z}\right\rangle$ is the thermal average of the $z$ component of the $\mathrm{Mn}^{2+}$ spin, given by the modified $5 / 2$ Brillouin function $(5 / 2) B_{5 / 2}\left(5 \mu_{\mathrm{B}} B / k_{\mathrm{B}} T_{\text {eff }}\right)$, and $T_{\text {eff }}=T+T_{0}$ is the effective temperature with $T_{0}$ the Mn-Mn interaction at $T=0 \mathrm{~K}$ determined from the macroscopic magnetizations [18]. In the effective-mass approximation the Hamiltonian of an electron is given by

$$
H=(\mathbf{p}+e \mathbf{A})^{2} / 2 m^{*}+V_{\mathrm{s}}+V_{\sigma_{z}}(z)-e E_{\mathrm{a}} z,
$$

where $\mathbf{p}$ is the momentum operator and $\mathbf{A}$ the vector potential. Notice that we include the effect of the magnetic field on the electron orbit which is often neglected in treatments of DMS materials. Further, $V_{\mathrm{s}}=g_{\mathrm{s}} \mu_{\mathrm{B}} \sigma_{z} B / 2$, with $g_{\mathrm{s}}$ the effective Landé factor and $\mu_{\mathrm{B}}$ the Bohr magneton, describes the Zeeman splitting, $E_{\mathrm{a}}$ is an electric field applied perpendicular to the quantum well, and $W$ is the width of this well. We will refer to the sum of the first two potential terms as the effective potential $V(z, B)$ given by

$$
V(z, B)=V_{\mathrm{s}}+V_{\sigma_{z}}(z) \text {. }
$$

\subsection{Perpendicular magnetic field $\mathbf{B}=B \hat{z}$}

When the magnetic field is along the $z$ axis the electron motion is decoupled from that in the $(x, y)$ plane. The in-plane motion is quantized in Landau orbits with wavefunction $F_{n}(x, y)$ and energies $E_{n}=\hbar \omega_{\mathrm{c}}(n+1 / 2)$, where $n$ is an integer $n=0,1,2, \ldots$ and $\omega_{\mathrm{c}}$ the cyclotron frequency. Then the Schrödinger equation $H \psi=E \psi$, with $\psi=F_{n}(x, y) \varphi(z)$ the wavefunction, reduces to the one-dimensional differential equation

$$
\left[-\frac{\hbar^{2}}{2 m^{*}} \frac{\mathrm{d}^{2}}{\mathrm{~d} z^{2}}+V(z, B)-e E_{\mathrm{a}} z\right] \varphi(z)=E_{z} \varphi(z),
$$

and the total energy is given by $E=E_{n}+E_{z}$. It is convenient to use the magnetic length $\ell_{\mathrm{B}}=(\hbar / e B)^{1 / 2}$ as the unit of length and $E_{*}=\hbar^{2} / 2 m^{*} \ell_{\mathrm{B}}^{2}=\hbar \omega_{\mathrm{c}} / 2$ as the unit of energy. Then equation (3) is rewritten as

$$
\left[-\frac{\mathrm{d}^{2}}{\mathrm{~d} z^{2}}+V(z, B)-\frac{V_{\mathrm{a}} z}{W}\right] \varphi(z)=E_{z} \varphi(z),
$$

where $V_{\mathrm{a}}=E_{\mathrm{a}} W$ is the potential drop over the quantum well due to the applied electric field.

\subsection{In-plane magnetic field $\mathbf{B}=B \hat{y}$}

Using the Landau gauge $\mathbf{A}=(B z, 0,0)$ the Schrödinger equation takes the form

$$
\left[\left(p_{y}^{2}+p_{z}^{2}\right) / 2 m^{*}+\left(p_{x}+e B z\right)^{2} / 2 m^{*}+V(z, B)-e E_{\mathrm{a}} z-E\right] \psi(x, y, z)=0 .
$$

Since the components $p_{y}$ and $p_{x}$ commute with $H$, the wavefunction is sought in the form

$$
\psi(x, y, z)=\mathrm{e}^{\mathrm{i} k_{x} x} \mathrm{e}^{\mathrm{i} k_{y} y} \varphi_{n, k_{x}}(z) / S^{1 / 2},
$$




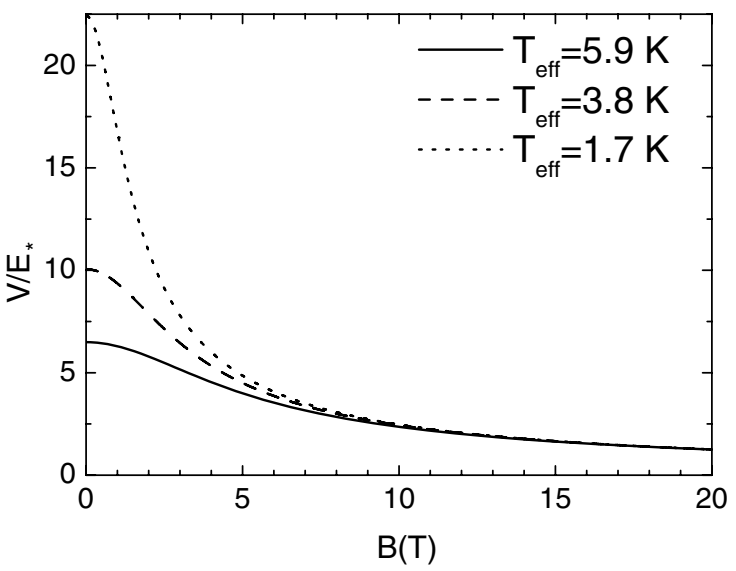

Figure 2. The potential step $V$ between the magnetic and non-magnetic layer, normalized to $E_{\star}=\hbar \omega_{\mathrm{c}} / 2$, as a function of the magnetic field $B$ for three different effective temperatures $T_{\text {eff }}=T+T_{0}$.

Table 1. Magnetic-field dependence of the potential step shown in figure 1.

\begin{tabular}{lllllllllll}
\hline$B(T)$ & 2 & 4 & 6 & 8 & 10 & 12 & 14 & 16 & 18 & 20 \\
\hline$V(\mathrm{meV})$ & 4.19 & 6.57 & 7.68 & 8.23 & 8.54 & 8.73 & 8.85 & 8.94 & 9.01 & 9.06 \\
\hline
\end{tabular}

where $S$ is the area in the $(x, y)$ plane. With this wavefunction the energy can be written as $E=E_{n}\left(k_{x}, k_{y}\right)=E_{n}\left(k_{x}\right)+\hbar^{2} k_{y}^{2} / 2 m^{*}$ and $\varphi_{n, k_{x}}(z)$ is the solution, in the dimensionless units specified above, of the equation

$$
\left[-\frac{\mathrm{d}^{2}}{\mathrm{~d} z^{2}}+\left(k_{x}+z\right)^{2}+V(z, B)-\frac{V_{\mathrm{a}} z}{W}-E_{n}\left(k_{x}\right)\right] \varphi_{n, k_{x}}(z)=0 .
$$

Equations (4) and (7) are solved numerically using standard finite-difference techniques and the results are presented below.

\section{Effect of a magnetic field in the absence of an external electric field}

The parameters we used in all calculations are $m^{*}=0.16 m_{\mathrm{e}}$, with $m_{\mathrm{e}}$ the free-electron mass, and $g_{\mathrm{s}}=1.1$. For $x=0.05$ we have $N_{0} \alpha=0.26 \mathrm{eV}$ and $T_{0}=1.7 \mathrm{~K}$ in the $\mathrm{Zn}_{1-x} \mathrm{Mn}_{x} \mathrm{Se}$ layer. In figure 2 we show the magnetic-field tunability of the size of the potential step $V$ in the quantum well (cf figure 1) between the $\mathrm{ZnSe}$ and the DMS material $\mathrm{Zn}_{1-x} \mathrm{Mn}_{x} \mathrm{Se}$ for $x=0.05$ and different values of the effective temperature. Notice that in figure 2 the potential step $V$ is scaled by $E_{*} \sim B$ which is responsible for the decrease of $V / E_{\star}$ as a function of $B$. In real units though $V$ increases with $B$ (see table 1). The absolute values of the effective potential $V$ in this table are given for $W=40 \mathrm{~nm}$ and $T_{\text {eff }}=5.9 \mathrm{~K}$. The magnitude of $V$ depends on $B$. For sufficiently strong $B$, increasing $B$ does not affect $\left\langle S_{z}\right\rangle$; only the Zeeman term is affected but not in a significant way. The results pertaining to sections 2.1 and 2.2 will be referred to, respectively, as case I and case II results. The zero of the energy is at the bottom of the well in the ZnSe layer and the Zeeman term is neglected because it is very small compared to the exchange splitting.

For case I the eigenvalues for the lowest 20 spin-down levels, with $k_{x}=k_{y}=0$, are shown in figure 3. The kink in the results near $n^{\prime}=10$ occurs when $E=0$. For $E<0$ the electron 


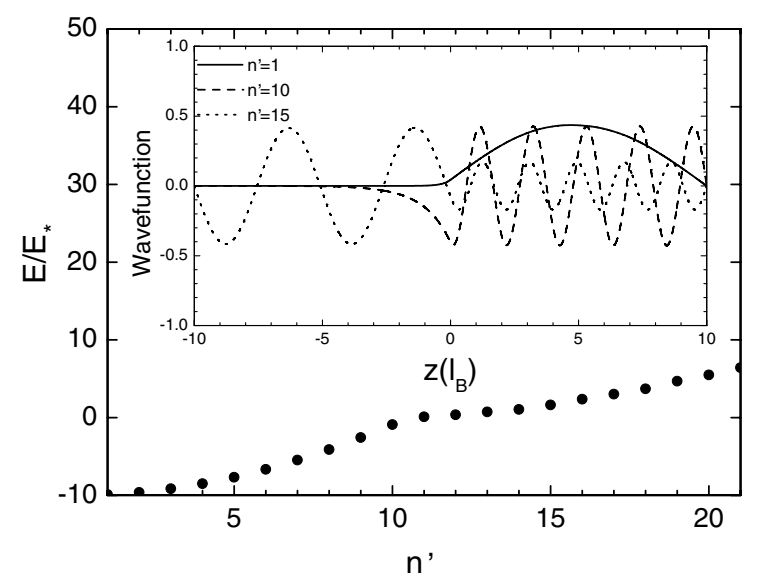

Figure 3. Energy spectrum versus quantum number $n^{\prime}$ for the first 20 spin-down levels, with $k_{x}=k_{y}=0$; the magnetic field is perpendicular to the quantum well. The height of the potential step is $V / E_{*}=10$ and the width of the well $W / \ell_{\mathrm{B}}=20$. The widths of the non-magnetic and magnetic layers are equal. The inset shows the wavefunctions for $n^{\prime}=1,10,15$.

is localized in the local potential well, while for $E>0$ it is localized in the broad quantum well. Thus the effective width of the potential well for $n^{\prime}<10$ is smaller than for $n^{\prime}>10$ and consequently the spacing between the energy levels is rather different. The wavefunctions of the ground and excited states for $n^{\prime}=1,10,15$ are shown in the inset. Due to the symmetric confinement the wavefunctions of the spin-up and spin-down electrons can be transformed into each other through a reflection with respect to $z=0$, because for the spin-up electrons the $\mathrm{ZnSe}$ layer plays the role of the well while for the spin-down ones the $\mathrm{Zn}_{1-x} \mathrm{Mn}_{x}$ Se layer does it. The ground state is quite localized in the well but the excited states can penetrate into the barrier and the $n^{\prime}=15$ state, with an energy larger than the height of the ZnSe barrier, is more localized in the barrier region.

From now on we take the magnetic field parallel to the interfaces, i.e. we consider case II. The dispersion relation is shown in figure 4 for the ten lowest levels for spin-up (dashed curves) and spin-down (solid curves) electrons, and two different widths $W$ of the quantum well as indicated. Notice that the spectrum is continuous but the energy is not symmetric in $k_{x}$. As can be seen, the energy levels of the spin-down electrons are lower than those of the spin-up electrons for $k_{x}<k_{x}^{\star}$, where the value of $k_{x}^{\star}$ depends on the system parameters and the strength of the magnetic field. For $k_{x}>k_{x}^{\star}$ both levels are degenerate. The difference between the minimal values of both dispersion curves is approximately equal to the height of the effective potential.

In order to understand the peculiar behaviour of the dispersion relation we consider the situation of figure 4(b). In the presence of a magnetic field the energy at low $\left|k_{x}\right|$ values can no longer be described by a parabola. The energy of the ground state of the spin-up electrons decreases to $E_{0} / E_{\star}=1$ with decreasing $\left|k_{x}\right|$, whereas the minimum of the energy curve for the spin-down electrons is at $E_{0} / E_{\star}=-9$. The cause of this behaviour is as follows. The energy of the lowest Landau level is +1 in our dimensionless units and therefore the bottom of the lowest, spin-down Landau level, which is in the right part of the well, becomes, in these units, $E_{0} / E_{\star}=-10+1=-9$. Similarly, the level at $E_{0} / E_{\star}=11$ can be understood as the lowest Landau level for the spin-up state localized in the right half of the quantum well. The right flat part of $E_{0}$ is the lowest Landau level in the left part of the quantum well. Comparing the 


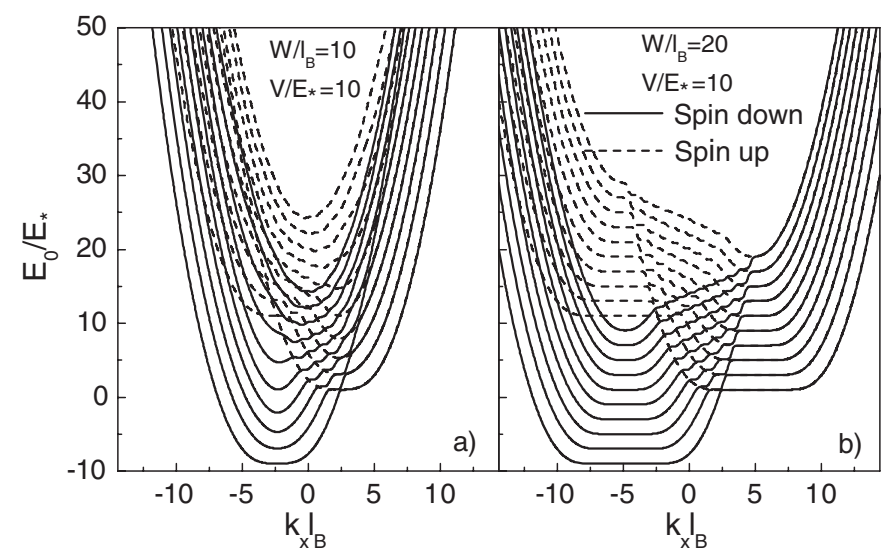

Figure 4. Energies of the ten lowest levels for spin-up (dashed curves) and spin-down (solid curves) electrons versus $k_{x} \ell_{\mathrm{B}}$, with $k_{y}=0, V / E_{*}=10$, and two different values of the well width: $W / \ell_{\mathrm{B}}=10$ in (a) and $W / \ell_{\mathrm{B}}=20$ in (b). The magnetic field is parallel to the quantum well.

two graphs, i.e. figures 4(a) and (b), it can be clearly seen that the dispersion relations behave similarly, but when the width of the layer decreases the energy increases more rapidly for fixed $k_{x}$.

The apparent steps in the dispersion relation seen in figures 4(a) and (b) can be understood as follows. It can be seen that with increasing $k_{x}$ the energies of the spin-up and spin-down electrons become equal; this results in an anti-crossing of energy levels. This anti-crossing occurs at higher values of $k_{x}$ as the energy of the state increases. It is also clearly seen that there is no step in the ground state level whereas there are $m$ steps in the $m$ th excited level. The reason is that for each spin the system in figure 1 can be viewed as two coupled square wells. Each square well has a set of well-defined energy levels and dispersions, and the two sets hybridize. The numerically exact calculation of the energy levels shows this hybridization that results in the anti-crossings of the different energy levels. Now it is clear that the discontinuouslike behaviour of the dispersion relation is a result of two overlapping sets of energy levels. This corresponds, respectively, to a dominant localization of the electron in the well $\left(k_{x}<0\right.$ for spin down) or in the barrier $\left(k_{x}>0\right.$ for spin up).

It is interesting to calculate the electron velocity $v_{x}=(1 / \hbar)\left(\partial E / \partial k_{x}\right)$ which is important for transport. The velocities for the lowest three spin-up and spin-down levels are shown in figure 5. Notice that the velocities vanish over a wide range of $k_{x}$ values separated by a region where the anti-crossings occur. For large $\left|k_{x}\right|$ values this is no longer the case and these states are replaced by the edge states. Notice also that the velocities of the spin-up and spin-down electrons have opposite signs and their absolute values are symmetric with respect to $k_{x}=0$. This behaviour of the velocity should be reflected, e.g., in the diffusive conductivity $\sigma_{x x}$ along the quantum well since $\sigma_{x x}$, at very low temperatures, is mainly determined by the value of $v_{x}^{2}$ at the Fermi level.

The DOS, $D(E)=\langle\delta(E-H)\rangle=1 /\left(\partial E / \partial k_{x}\right)$, is shown in figure 6. It shows the well-known $1 /\left(E-E_{0}\right)^{1 / 2}$ singularities for $1 \mathrm{D}$ systems each time a new subband is occupied. When the Fermi energy is small it is clear that only electrons with spin-down polarization are present. This results in a strongly spin-polarized current along the quantum well. Only when the electron density is sufficiently large and the Fermi energy is positive $\left(E_{\mathrm{F}}>0\right)$ can electrons occupy the spin-up state. 


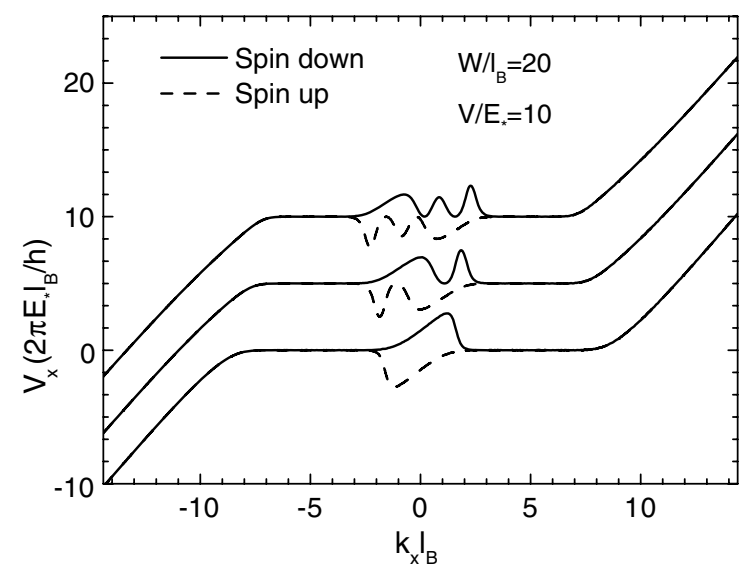

Figure 5. Velocity versus $k_{x} \ell_{\mathrm{B}}$ for the three lowest spin-up and spin-down levels. For clarity the curves for the second and third levels are shifted up by 5 and 10 units, respectively. The magnetic field is parallel to the quantum well.

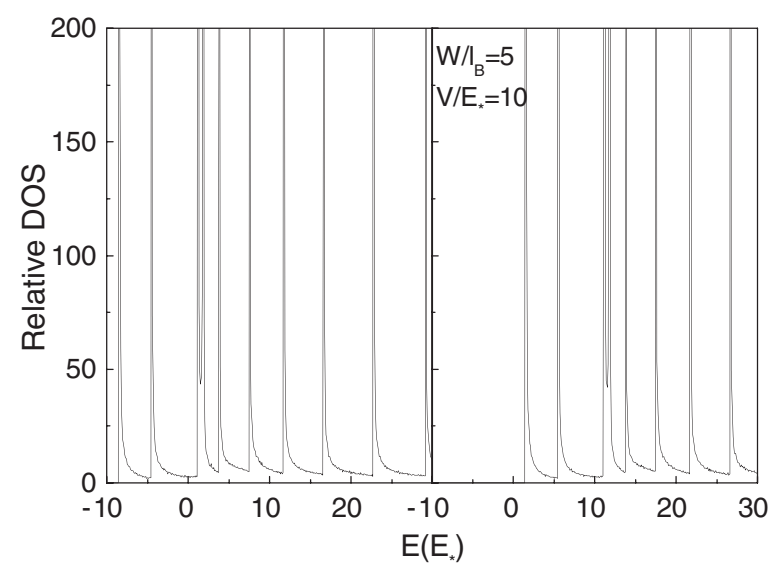

Figure 6. The density of states (DOS) versus energy for spin-down electrons (left panel) and spinup electrons (right panel) for $W / \ell_{\mathrm{B}}=5$ and $V / E_{*}=10$. The magnetic field is parallel to the quantum well.

\section{Effect of an electric field}

The dispersion relation of the ground state and of the first, third, fifth and seventh excited states is shown in figure 7 for two different biases $V_{\mathrm{a}}$. As mentioned earlier, the Zeeman energy is negligible compared to the exchange splitting and was not taken into account. It is seen that applying a positive bias shifts the energy levels upward, in particular for $k_{x}<0$. If the bias is negative, the levels are shifted downward. Thus, one can spatially shift the polarized electrons from the DMS material into the non-DMS material by applying an electric field. In the non-DMS material we have unpolarized electrons because the spin-down and spin-up levels coincide. This is clearly seen for larger positive $V_{\mathrm{a}}$ values for which the absolute minimum of the energy spectrum occurs at positive $k_{x}$ values. The quantum well for a given spin is spatially asymmetric because of the external magnetic field. The carriers have higher density in the deeper part of the well. By applying an electric field the deeper part can be raised above the 


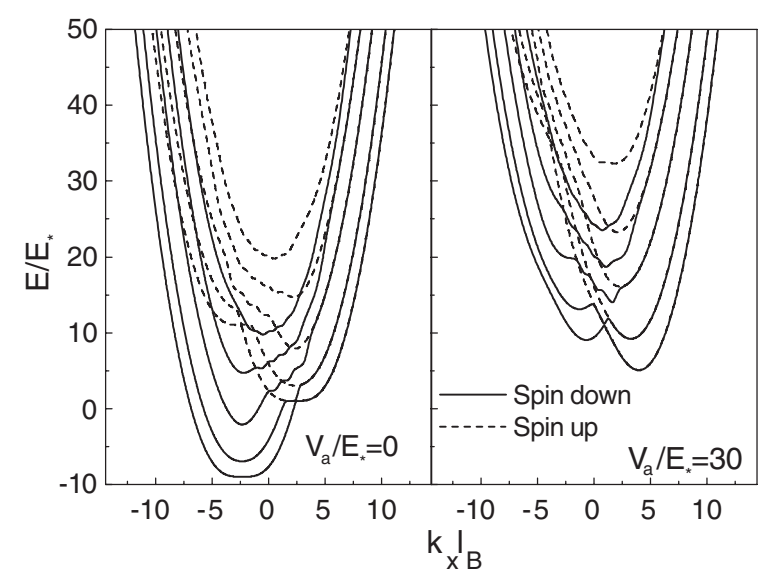

Figure 7. Dispersion relation of the ground state and of the first, third, fifth and seventh excited spin-up and spin-down states versus $k_{x} \ell_{\mathrm{B}}$ for two different values of $V_{\mathrm{a}} / E_{*}$ with $W / \ell_{\mathrm{B}}=5$ and $V / E_{*}=10$. The magnetic field is parallel to the quantum well.

shallower part of the well (namely, to reverse the asymmetry). Then the spatial density of the carriers will move accordingly. In our case this field-charge interaction phenomenon can be used to manipulate the spatial density of the different spins. Thus, by using an electric field we can tune the relative population of the spin-up and spin-down electrons and consequently control the magnetization of the electron system. Using an electric field we can depolarize the 2DEG in the quantum well.

The density of states of the spin-up and spin-down levels is shown in figure 8 for two values of $V_{\mathrm{a}}$. The plot clearly shows the tunability of the states by the electric field.

\section{Concluding remarks}

We studied the energy spectrum, velocity and density of states of an electron in confined nonmagnetic/magnetic heterostructures. The resulting quantum well has a potential step at the interface between the non-magnetic and magnetic material and, depending on its height, can strongly affect these electron properties. As a concrete example we studied in detail these properties for an electron in a $\mathrm{ZnSe} / \mathrm{Zn}_{1-x} \mathrm{Mn}_{x}$ Se heterostructure, shown in figure 1, confined by infinitely high walls. Our study took into account a magnetic field, perpendicular or parallel to the well, as well as an electric field perpendicular to the well. The assumption of infinitely high barriers is not essential for the physics discussed in this study and may easily be relaxed to that of finite-height barriers.

The potential step mentioned above leads to a spatial separation of spin-up and spin-down electrons. We obtained a rich band structure shown in figures 3, 4 and 7, an unusual group velocity for the electrons (figure 5) as well as a density of states with an unusual structure (figures 6 and 8). As shown in section 4, this band structure can be tuned by a perpendicular electric field. It is worth noting how the spin state can be manipulated by such an electric field as figures 6-8 demonstrate. As detailed in section 4, this manipulation is due to an electric-field induced spatial shift of the electron wavefunction at constant electron density. It is obvious that similar results should be obtained for confined non-magnetic/magnetic heterostructures other than the particular one studied in the present work. It should be noticed though that we have neglected the positional disorder of Mn atoms on the subbands [12, 13]. For low concentrations 


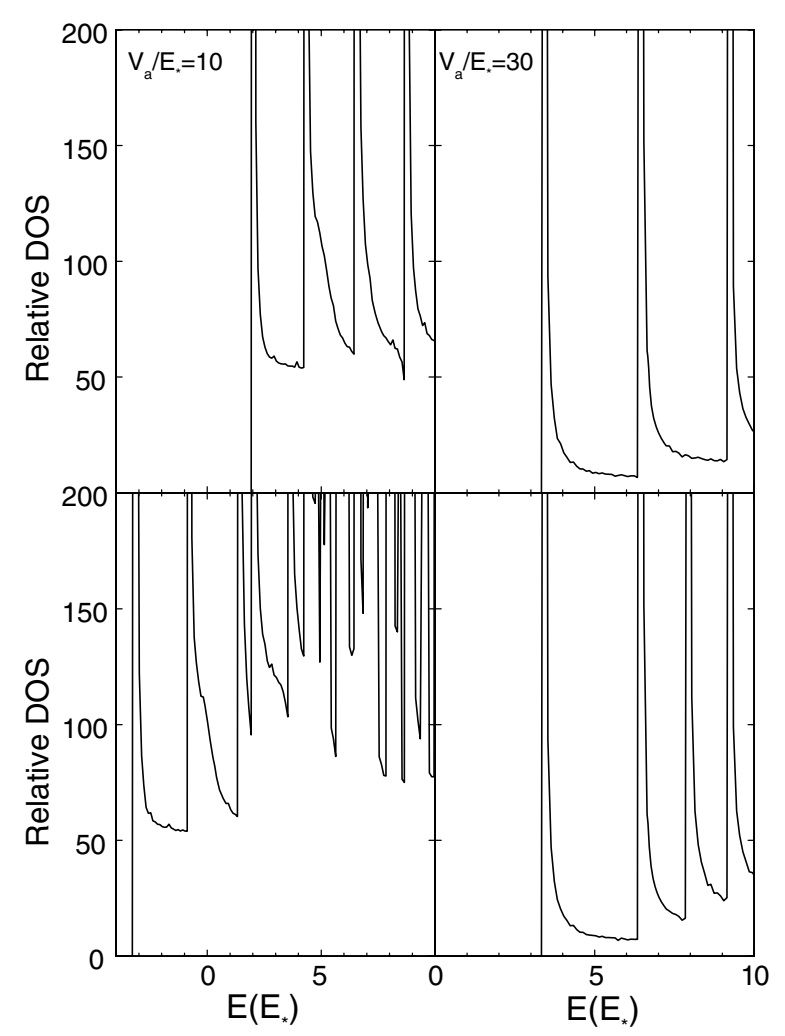

Figure 8. Relative density of states versus energy for spin-down (bottom panels) and spin-up electrons (top panels) for biases, $V_{\mathrm{a}} / E_{*}=10$ (left panels) and $V_{\mathrm{a}} / E_{*}=30$ (right panels) with $W / \ell_{\mathrm{B}}=20$ and $V / E_{*}=10$. The magnetic field is parallel to the quantum well.

$x$ this can have an important effect on the subbands and the magnetic properties but we expect it will not invalidate our conclusions qualitatively.

Though we did not evaluate the transport coefficients, it is evident, especially on account of the electron velocities shown in figure 5, that transport in such a non-magnetic/magnetic heterostructure will be strongly affected by the presence of the potential step and the confinement producing the edge states. As indicated at the end of section 3, a strongly spinpolarized current can result along the quantum well. This step and confinement should also affect the spin polarization. The pertinent transport study will be undertaken separately.

\section{Acknowledgments}

This work was supported by the Flemish-Hungarian bilateral programme, the Canadian NSERC grant no. OGP0121756, and the Flemish Science Foundation (FWO-Vl). SB was supported by the EU Marie Curie training project: HPTM-CT-2001-00394.

\section{References}

[1] Winkler R 2003 Spin-Orbit Coupling Effects in Two-Dimensional Electron and Hole Systems (Springer Tracts in Modern Physics vol 191) (Berlin: Springer) 
Wolf S A et al 2001 Science 2941488

[2] Matulis A, Peeters F M and Vasilopoulos P 1994 Phys. Rev. Lett. 721518

Reijniers J and Peeters F M 2000 J. Phys.: Condens. Matter 129771

Papp G and Peeters F M 2004 J. Phys.: Condens. Matter 168275

[3] Peeters F M and De Boeck J 2002 Nanostructured Materials and Nanotechnology ed N S Nalwa

(New York: Academic) p 247

Dietl T 2003 Advances in Solid State Physics ed B Kramer (Berlin: Springer) p 413

[4] Gould C, Slobodskyy A, Slobodskyy T, Grabs P, Becker C R, Schmidt G and Molenkamp L W 2004 Phys. Status Solidi b 241700

[5] Guo Y, Gu B-L, Wang H and Kawazoe Y 2001 Phys. Rev. B 63214415

Chang K and Peeters F M 2001 Solid State Commun. 120181

[6] Wang X F and Vasilopoulos P 2002 Appl. Phys. Lett. 811636

[7] Papp G, Borza S and Peeters F M 2005 J. Appl. Phys. 97113901

Papp G, Borza S and Peeters F M 2006 Phys. Status Solidi b 2431956

[8] Chang K and Peeters F M 2003 Phys. Rev. B 68205320

[9] Ghali M, Kossut J, Janik E, Teppe F and Scalbert D 2004 Phys. Status Solidi b 241688

[10] Heimbrod W, Gridneva L, Happ M, Hoffmann N, Rabe M and Henneberger F 1998 Phys. Rev. B 581162

[11] Boselli M A, Ghazali A and da Cunha Lima I C 2000 Phys. Rev. B 628895

[12] Berciu M and Bhatt R N 2001 Phys. Rev. Lett. 87107203

[13] Das Sarma S, Hwang E H and Kaminski A 2003 Phys. Rev. B 67155201

[14] Egues J C 1998 Phys. Rev. Lett. 804578

[15] Dai N, Luo H, Zhang F C, Samarth N, Dobrowolska M and Furdyna J K 1994 Phys. Rev. Lett. 673824

[16] Zutic I, Fabian J and Das Sarma S 2004 Rev. Mod. Phys. 76323

[17] Chiba D, Yamanouch M, Matsukura F and Ohno H 2003 Science 301943

[18] Twardowski A, Von Ortenberg M, Demianuk M and Pauthenet R 1984 Solid State Commun. 51849 